5th International Workshop on Astronomy and

Relativistic Astrophysics (IWARA2011)

International Journal of Modern Physics: Conference Series

Vol. 18 (2012) 164-173

(C) World Scientific Publishing Company

DOI: $10.1142 / \mathrm{S} 2010194512008392$

\title{
QUANTUM EFFECTS IN HIGHER-DIMENSIONAL COSMOLOGICAL MODELS
}

\author{
ARAM A. SAHARIAN \\ Department of Physics, Yerevan State University \\ 1 Alex Manoogian Street, 0025 Yerevan, Armenia \\ saharian@ysu.am
}

\begin{abstract}
Vacuum energy density and stresses are investigated for a scalar field in de Sitter spacetime with an arbitrary number of toroidally compactified spatial dimensions and in antide Sitter spacetime with two parallel branes. On the branes the field obeys the Robin boundary conditions. The behavior of the vacuum expectation values is discussed in various asymptotic regions of the parameters. Applications are given to Randall-Sundrum type braneworlds.
\end{abstract}

Keywords: de Siter spacetiem; anti-de Sitter spacetiem; Casimir effect.

PACS numbers: 04.62.+v, 04.50.-h, 11.10.Kk, 04.20.Gz

\section{Introduction}

A number of high-energy theories of fundamental physics are formulated in higherdimensional spacetimes. In particular, the idea of extra dimensions has been used in supergravity and superstring theories. Two types of models with extra dimensions were discussed in the literature. The first one corresponds to Kaluza-Klein type models, where it is assumed that the extra dimensions are compactified and all of them are accessable for the standard model fields. In the second type of models, the standard model fields are localized on a 3-dimensional hypersurface (brane) and the extra dimensions are accessable for the gravitational and for some additional hypothetical fields. These models provide a solution to the hierarchy problem between the gravitational and electroweak mass scales ${ }^{1,2}$. The main idea to resolve the large hierarchy is that the small coupling of four dimensional gravity is generated by the large physical volume of extra dimensions. These theories provide a novel setting for discussing phenomenological and cosmological issues related to extra dimensions.

In both types of models with extra dimensions the boundary conditions imposed on possible field configurations change the spectrum of the vacuum fluctuations and lead to the Casimir-type contributions to the vacuum expectation values of physical observables. The Casimir effect (for a review see Ref. 3) has been used as a stabilization mechanism for moduli fields parametrizing the size of the extra 
dimensions. The Casimir energy coming from the extra dimensions can also serve as a model for dark energy (see Ref. 4 and references therein). In the first part of the present talk, based on Refs. 5, 6, we describe the effects of the toroidal compactification of spatial dimensions in de Sitter (dS) spacetime on the properties of quantum vacuum for a scalar field with general curvature coupling parameter. The corresponding one-loop quantum effects for a fermionic field are studied in Refs. 7, 8. The second part, based on Refs. 9, 10, is devoted to the investigation of the vacuum energy-momentum tensor for a scalar field in the geometry of two parallel branes in anti-de Sitter (AdS) spacetime. This geometry is employed in the Randall-Sundrum type braneworld models ${ }^{2}$.

\section{2. dS spacetime with totoidally compact dimensions}

Consider a free scalar field with curvature coupling parameter $\xi$. The corresponding field equation has the form

$$
\left(\nabla_{l} \nabla^{l}+m^{2}+\xi R\right) \varphi=0
$$

where $R$ is the Ricci scalar for the background spacetime. For minimally and conformally coupled scalars one has $\xi=0$ and $\xi=\xi_{c}=(D-1) / 4 D$ correspondingly. In this section, as a background geometry we consider $(D+1)$-dimensional dS spacetime generated by a positive cosmological constant $\Lambda$. In planar coordinates the corresponding line element has the form

$$
d s^{2}=d t^{2}-e^{2 t / \alpha} \sum_{l=1}^{D}\left(d x^{l}\right)^{2},
$$

with the parameter $\alpha^{2}=D(D-1) /(2 \Lambda)$. For this geometry $R=D(D+1) / \alpha^{2}$. We assume that the spatial coordinate $x^{l}, l=p+1, \ldots, D$, is compactified to $\mathrm{S}^{1}$ of the length $L_{l}: 0 \leqslant x^{l} \leqslant L_{l}$, and for the other coordinates we have $-\infty<x^{l}<+\infty$, $l=1, \ldots, p$. Hence, we consider the spatial topology $\mathrm{R}^{p} \times\left(\mathrm{S}^{1}\right)^{q}$, where $q=D-p$. Along the compact dimensions we impose the periodicity conditions

$$
\varphi\left(t, x^{1}, \ldots, x^{p}, x^{p+1}, \ldots, x^{l}+L_{l}, \ldots, x^{D}\right)= \pm \varphi\left(t, x^{1}, \ldots, x^{p}, x^{p+1}, \ldots, x^{l}, \ldots, x^{D}\right),
$$

where $l=p+1, \ldots, D$, and the upper/lower sign corresponds to untwisted/twisted scalar field.

As a result of boundary conditions, the spectrum of the vacuum fluctuation of the field $\varphi$ is modified compared with the case of $\mathrm{dS}$ spacetime with trivial topology $\mathrm{R}^{D}$. This leads to the change of the vacuum expectation values (VEVs) for physical observables (topological Casimir effect). We are interested in the VEV of the energymomentum tensor. This quantity acts as a source of gravity in the semiclassical Einstein equations and plays an important role in modelling self-consistent dynamics involving the gravitational field. Assuming that the field is prepared in the BunchDavies vacuum state ${ }^{11}$, we have the following recurrence relation for the VEV of 
the energy-momentum tensor

$$
\left\langle T_{i}^{k}\right\rangle_{p, q}=\left\langle T_{i}^{k}\right\rangle_{p+1, q-1}+\Delta_{p+1}\left\langle T_{i}^{k}\right\rangle_{p, q}
$$

Here $\left\langle T_{i}^{k}\right\rangle_{p+1, q-1}$ is the VEV for dS spacetime with topology $\mathrm{R}^{p+1} \times\left(\mathrm{S}^{1}\right)^{q-1}$ and the part $\Delta_{p+1}\left\langle T_{i}^{k}\right\rangle_{p, q}$ is induced by the compactness along the $x^{p+1}$ - direction.

For the corresponding energy density one has

$$
\begin{aligned}
\Delta_{p+1}\left\langle T_{0}^{0}\right\rangle_{p, q}= & \frac{2 \eta^{D} L_{p+1}}{(2 \pi)^{(p+3) / 2} V_{q} \alpha^{D+1}} \sum_{n=1}^{\infty}( \pm 1)^{n} \sum_{\mathbf{n}_{q-1}} \int_{0}^{\infty} d x \\
& \times \frac{x F_{\nu}^{(0)}(x \eta)}{\left(n L_{p+1}\right)^{p-1}} f_{(p-1) / 2}\left(n L_{p+1} \sqrt{x^{2}+k_{\mathbf{n}_{q-1}}^{2}}\right),
\end{aligned}
$$

where $V_{q}=L_{p+1} \cdots L_{D}$ is the volume of the compact subspace, $\sum_{\mathbf{n}_{q-1}}=$ $\sum_{n_{p+2}=-\infty}^{+\infty} \cdots \sum_{n_{D}=-\infty}^{+\infty}, f_{\mu}(y)=y^{\mu} K_{\mu}(y), K_{\mu}(y)$ is the MacDonald function and

$$
\begin{aligned}
F_{\nu}^{(0)}(y)= & y^{2}\left[I_{-\nu}^{\prime}(y)+I_{\nu}^{\prime}(y)\right] K_{\nu}^{\prime}(y)+D(1 / 2-2 \xi) y G_{\nu}^{\prime}(y) \\
& +G_{\nu}(y)\left(\nu^{2}+2 m^{2} \alpha^{2}-y^{2}\right)
\end{aligned}
$$

with $I_{\nu}(y)$ being the modified Bessel function, $\nu=\sqrt{D^{2} / 4-D(D+1) \xi-m^{2} \alpha^{2}}$ and

$$
G_{\nu}(y)=\left[I_{-\nu}(y)+I_{\nu}(y)\right] K_{\nu}(y) .
$$

In (5), the upper/lower sign corresponds to untwisted/twisted scalar field,

$$
k_{\mathbf{n}_{q-1}}^{2}=\sum_{l=p+2}^{D}\left[2 \pi\left(n_{l}+g_{l}\right) / L_{l}\right]^{2},
$$

where $g_{l}=0$ for untwisted scalar and $g_{l}=1 / 2$ for twisted scalar field.

The vacuum stresses are presented in the form (no summation over $i$ )

$$
\begin{aligned}
\Delta_{p+1}\left\langle T_{i}^{i}\right\rangle_{p, q}= & \frac{2 \eta^{D} L_{p+1}}{(2 \pi)^{(p+3) / 2} V_{q} \alpha^{D+1}} \sum_{n=1}^{\infty} \frac{( \pm 1)^{n}}{\left(n L_{p+1}\right)^{p-1}} \sum_{\mathbf{n}_{q-1}} \int_{0}^{\infty} d x x \\
& \times\left[F_{\nu}(x \eta) f_{(p-1) / 2}\left(n L_{p+1} \sqrt{x^{2}+k_{\mathbf{n}_{q-1}}^{2}}\right)\right. \\
& \left.-\frac{2 G_{\nu}(x \eta)}{\left(n L_{p+1} / \eta\right)^{2}} f_{p}^{(i)}\left(n L_{p+1} \sqrt{x^{2}+k_{\mathbf{n}_{q-1}}^{2}}\right)\right]
\end{aligned}
$$

where we have introduced the notations

$$
\begin{aligned}
f_{p}^{(i)}(y)= & f_{(p+1) / 2}(y), i=1, \ldots, p, f_{p}^{(p+1)}(y)=-y^{2} f_{(p-1) / 2}(y)-p f_{(p+1) / 2}(y), \\
f_{p}^{(i)}(y)= & {\left[2 \pi n\left(n_{i}+g_{i}\right) L_{p+1} / L_{i}\right]^{2} f_{(p-1) / 2}(y), i=p+2, \ldots, D } \\
F_{\nu}(y)= & {[2(D+1) \xi-D / 2] y G_{\nu}^{\prime}(y)+(4 \xi-1) y^{2} K_{\nu}^{\prime}(y)\left[I_{-\nu}^{\prime}(y)+I_{\nu}^{\prime}(y)\right] } \\
& +G_{\nu}(y)\left[(4 \xi-1)\left(y^{2}+\nu^{2}\right)\right] .
\end{aligned}
$$

As it is seen from the obtained formulas, the topological parts in the VEVs are timedependent and, hence, the local dS symmetry is broken by the non-trivial topology. 
By taking into account the relation between the conformal and synchronous time coordinates, we see that the VEV of the energy-momentum tensor is a function of the combination $L_{l} / \eta=L_{l} e^{t / \alpha} / \alpha$, which is the comoving length of the compactified dimension measured in units of the dS curvature scale.

The recurring application of formula (4) allows us to write the VEV in the form

$$
\left\langle T_{i}^{k}\right\rangle_{p, q}=\left\langle T_{i}^{k}\right\rangle_{\mathrm{dS}}+\left\langle T_{i}^{k}\right\rangle_{\mathrm{c}},\left\langle T_{i}^{k}\right\rangle_{\mathrm{c}}=\sum_{l=1}^{q} \Delta_{D-l+1}\left\langle T_{i}^{k}\right\rangle_{D-l, l}
$$

where the part corresponding to uncompactified dS spacetime, $\left\langle T_{i}^{k}\right\rangle_{\mathrm{dS}}$, is explicitly decomposed. As a consequence of the maximal symmetry for dS spacetime and the Bunch-Davies vacuum state, the latter has the structure $\left\langle T_{i k}\right\rangle_{\mathrm{dS}}=$ const $\cdot g_{i k}$. The part $\left\langle T_{i}^{k}\right\rangle_{\mathrm{c}}$ is induced by the comactness of the $q$-dimensional subspace. This part is finite and the renormalization is needed for the uncompactified dS part only. We could expect this result, since the local geometry is not changed by the toroidal compactification.

For a conformally coupled massless scalar field $\nu=1 / 2$ and we find (no summation over $i$ )

$$
\Delta_{p+1}\left\langle T_{i}^{i}\right\rangle_{p, q}=-\frac{2(\eta / \alpha)^{D+1} L_{p+1}}{(2 \pi)^{p / 2+1} V_{q}} \sum_{n=1}^{\infty}( \pm 1)^{n} \sum_{\mathbf{n}_{q-1} \in \mathbb{Z}_{q-1}} \frac{g_{p}^{(i)}\left(n L_{p+1} k_{\mathbf{n}_{q-1}}\right)}{\left(n L_{p+1}\right)^{p+2}}
$$

with the notations

$$
\begin{aligned}
& g_{p}^{(i)}(y)=f_{p / 2+1}(y), i=0,1, \ldots, p, g_{p}^{(p+1)}(y)=-(p+1) f_{p / 2+1}(y)-y^{2} f_{p / 2}(y), \\
& g_{p}^{(i)}(y)=\left[2 \pi n\left(n_{i}+g_{i}\right) L_{p+1} / L_{i}\right]^{2} f_{p / 2}(y), i=p+2, \ldots, D .
\end{aligned}
$$

In this case the topological part in the VEV of the energy-momentum tensor is traceless and the trace anomaly is contained in the uncompactified dS part only. Formula (13) could be obtained from the corresponding result in $(D+1)$-dimensional Minkowski spacetime with spatial topology $\mathrm{R}^{p} \times\left(\mathrm{S}^{1}\right)^{q}$, taking into account that two problems are conformally related: $\Delta_{p+1}\left\langle T_{i}^{k}\right\rangle_{p, q}=(\eta / \alpha)^{D+1} \Delta_{p+1}\left\langle T_{i}^{k}\right\rangle_{p, q}^{(\mathrm{M})}$.

For small values of the ratio, $L_{p+1} / \eta \ll 1$, to the leading order, $\Delta_{p+1}\left\langle T_{i}^{k}\right\rangle_{p, q}$ coincides with the corresponding result for a conformally coupled massless field, given by (13). For a fixed value of the ratio $L_{p+1} / \alpha$, this limit corresponds to $t \rightarrow-\infty$ and the topological part $\left\langle T_{i}^{k}\right\rangle_{\mathrm{c}}$ behaves like $\exp [-(D+1) t / \alpha]$. By taking into account that the part $\left\langle T_{i}^{k}\right\rangle_{\mathrm{dS}}$ is time independent, from here we conclude that in the early stages of the cosmological expansion the topological part dominates in the VEV of the energy-momentum tensor. In particular, in this limit the total energy density is negative.

For small values of the ratio $\eta / L_{p+1}$ and for real values of the parameter $\nu$ we have $\Delta_{p+1}\left\langle T_{0}^{0}\right\rangle_{p, q} \propto\left(\eta / L_{p+1}\right)^{D-2 \nu}$. The corresponding energy density is positive for a minimally coupled scalar field and for a conformally coupled massive scalar field. For the vacuum stresses, to the leading order, we have the relation (no summation over $i) \Delta_{p+1}\left\langle T_{i}^{i}\right\rangle_{p, q} \approx(2 \nu / D) \Delta_{p+1}\left\langle T_{0}^{0}\right\rangle_{p, q}, i=1, \ldots, D$, for $\eta / L_{p+1} \ll 1$. 
The coefficient in this relation does not depend on $p$ and it takes place for the total topological part of the VEV as well. Hence, in the limit under consideration the topological parts in the vacuum stresses are isotropic. Note that this limit corresponds to late times in terms of synchronous time coordinate $t,\left(\alpha / L_{p+1}\right) e^{-t / \alpha} \ll 1$, and the topological part in the VEV is suppressed by the factor $\exp [-(D-2 \nu) t / \alpha]$. As the uncompactified dS part is constant, it dominates the topological part at the late stages of the cosmological evolution.

For small values of the ratio $\eta / L_{p+1}$ and for purely imaginary $\nu$, the energy density and the vacuum stresses behave like $\Delta_{p+1}\left\langle T_{0}^{0}\right\rangle_{p, q} \propto e^{-D t / \alpha} \sin (2|\nu| t / \alpha+\phi)$ and $\Delta_{p+1}\left\langle T_{i}^{i}\right\rangle_{p, q} \propto e^{-D t / \alpha} \cos (2|\nu| t / \alpha+\phi)$. Hence, in the case under consideration, at late stages of the cosmological evolution the topological part is suppressed by the factor $\exp (-D t / \alpha)$ and the damping of the corresponding $\mathrm{VEV}$ is oscillatory.

\section{Vacuum densities for branes in anti-de Sitter spacetime}

In this section as a background geometry we consider $(D+1)$-dimensional AdS spacetime. This spacetime is one of the simplest and most interesting backgrounds allowed by general relativity. In Poincare coordinates the corresponding line element is given by

$$
d s^{2}=g_{i k} d x^{i} d x^{k}=e^{-2 y / \alpha} \eta_{\mu \nu} d x^{\mu} d x^{\nu}-d y^{2},
$$

where $\eta_{\mu \nu}$ is the metric for the $D$-dimensional Minkowski spacetime. For the corresponding Ricci scalar one has $R=-D(D+1) / \alpha^{2}$. By making a coordinate transformation $z=\alpha e^{y / \alpha}$, metric (15) is written in a conformally-flat form: $d s^{2}=(\alpha / z)^{2} \eta_{i k} d x^{i} d x^{k}$.

Below we will study quantum vacuum effects induced by two parallel infinite branes, located at $y=a$ and $y=b, a<b$. On the branes the field $\varphi$ obeys the Robin boundary conditions

$$
\left(\tilde{A}_{y}+\tilde{B}_{y} \partial_{y}\right) \varphi(x)=0, \quad y=a, b,
$$

with constant coefficients $\tilde{A}_{y}, \tilde{B}_{y}$. The presence of boundaries modifies the spectrum for the zero-point fluctuations of the scalar field under consideration. This leads to the modification of the VEVs of physical quantities compared with the case of AdS spacetime without branes. In particular, vacuum forces arise acting on the boundaries. Motivated by the applications to braneworld type scenarios, the vacuum energy in the two-branes setup has been considered in a large number of papers (see, for instance, references given in Refs. 12). Here we present the results for the VEV of the energy-momentum tensor.

\subsection{Casimir densities for a single brane}

In this section we will consider the VEV of the geometry of a single brane located at $y=a$. The corresponding vacuum energy-momentum tensor is diagonal and is 
presented in the form

$$
\left\langle 0\left|T_{i}^{k}\right| 0\right\rangle=\left\langle T_{i}^{k}\right\rangle_{\mathrm{AdS}}+\left\langle T_{i}^{k}\right\rangle^{(a)},
$$

where $\left\langle T_{i}^{k}\right\rangle_{\mathrm{AdS}}$ is the renormalized VEV for the energy-momentum tensor in the AdS background without boundaries, and the term $\left\langle T_{i}^{k}\right\rangle^{(a)}$ is induced by a single brane at $y=a$. The brane-induced part is finite for points away the brane and the renormalization procedure is needed for the boundary-free part only. The latter is well-investigated in the literature. Due to the maximal symmetry of AdS spacetime one has $\left\langle T_{i}^{k}\right\rangle_{\text {AdS }}=$ const $\cdot g_{i k}$. The boundary-induced part is given by the expressions

$$
\begin{aligned}
& \left\langle T_{i}^{k}\right\rangle^{(a)}=-\frac{\alpha^{-D-1} z^{D} \delta_{i}^{k}}{2^{D-1} \pi^{D / 2} \Gamma(D / 2)} \int_{0}^{\infty} d u u^{D-1} \frac{\bar{I}_{\sigma}^{(a)}\left(u z_{a}\right)}{\bar{K}_{\sigma}^{(a)}\left(u z_{a}\right)} F^{(i)}\left[K_{\sigma}(u z)\right], z>z_{a}, \\
& \left\langle T_{i}^{k}\right\rangle^{(a)}=-\frac{\alpha^{-D-1} z^{D} \delta_{i}^{k}}{2^{D-1} \pi^{D / 2} \Gamma(D / 2)} \int_{0}^{\infty} d u u^{D-1} \frac{\bar{K}_{\sigma}^{(a)}\left(u z_{a}\right)}{\bar{I}_{\sigma}^{(a)}\left(u z_{a}\right)} F^{(i)}\left[I_{\sigma}(u z)\right], z<z_{a},
\end{aligned}
$$

where $z_{j}=\alpha e^{j / \alpha}$ and $\sigma=\sqrt{(D / 2)^{2}-D(D+1) \xi+m^{2} \alpha^{2}}$. In (18), for a function $F(x)$ we have defined the barred notation

$$
\bar{F}^{(j)}(x)=A_{j} F(x)+B_{j} x F^{\prime}(x), \quad A_{j}=\tilde{A}_{j}+\tilde{B}_{j} D /(2 \alpha), \quad B_{j}=\tilde{B}_{j} / \alpha,
$$

and for a given function $g(v)$ the functions $F^{(i)}[g(v)]$ are given by

$$
\begin{aligned}
F^{(i)}[g(v)]= & \left(\frac{1}{2}-2 \xi\right)\left[v^{2} g^{\prime 2}(v)+\left(D+\frac{4 \xi}{4 \xi-1}\right) v g(v) g^{\prime}(v)+\right. \\
& \left.+\left(\sigma^{2}+v^{2}+\frac{2 v^{2}}{D(4 \xi-1)}\right) g^{2}(v)\right], \quad i=0,1, \ldots, D-1 \\
F^{(D)}[g(v)]= & -\frac{v^{2}}{2} g^{\prime 2}(v)+\frac{D}{2}(4 \xi-1) v g(v) g^{\prime}(v)+ \\
& +\frac{1}{2}\left[\sigma^{2}+v^{2}+2 \xi D(D+1)-D^{2} / 2\right] g^{2}(v) .
\end{aligned}
$$

We assume values of the curvature coupling parameter for which $\sigma$ is real. For imaginary $\sigma$ the ground state becomes unstable ${ }^{13}$. Note that VEVs (18) depend only on the ratio $z / z_{a}$ which is related to the proper distance from the plate by the equation $z / z_{a}=e^{(y-a) / \alpha}$. As we see, for the part of the energy-momentum tensor corresponding to the coordinates in the hyperplane parallel to the plates one has $\left\langle T_{\mu \nu}\right\rangle^{(a)} \propto \eta_{\mu \nu}$. Of course, we could expect this result from the problem symmetry.

For a conformally coupled massless scalar $\sigma=1 / 2$, and it can be seen that $\left\langle T_{i}^{k}\right\rangle^{(a)}=0$ in the region $z>z_{a}$ and

$$
\left\langle T_{D}^{D}\right\rangle^{(a)}=-D\left\langle T_{0}^{0}\right\rangle^{(a)}=-\frac{\alpha^{-D-1}\left(z / z_{a}\right)^{D+1}}{(4 \pi)^{D / 2} \Gamma(D / 2)} \int_{0}^{\infty} \frac{t^{D} d t}{\frac{B_{a}(t-1)+2 A_{a}}{B_{a}(t+1)-2 A_{a}} e^{t}+1}
$$

in the region $z<z_{a}$. Note that the corresponding energy-momentum tensor for a single Robin plate in the Minkowski bulk vanishes ${ }^{14}$ and the result for the region $z>z_{a}$ is obtained by a simple conformal transformation from that for the Minkowski case. In the region $z<z_{a}$ this procedure does not work as in the AdS problem one 
has $0<z<z_{a}$ instead of $-\infty<z<z_{a}$ in the Minkowski problem and, hence, the part of AdS under consideration is not a conformal image of the corresponding manifold in the Minkowski spactime.

In the limit $z \rightarrow z_{a}$ for a fixed $\alpha$ the expressions (18) diverge. Near the brane, to the leading order, we have $\left\langle T_{0}^{0}\right\rangle^{(a)} \propto\left|1-z_{a} / z\right|^{-D-1}$ and $\left\langle T_{D}^{D}\right\rangle^{(a)} \approx\left(1-z_{a} / z\right)\left\langle T_{0}^{0}\right\rangle^{(a)}$. In this region, the vacuum energy densities have opposite signs for the cases of Dirichlet $\left(B_{a}=0\right)$ and non-Dirichlet $\left(B_{a} \neq 0\right)$ boundary conditions. For a conformally coupled massless scalar the vacuum energy-momentum tensor vanishes in the region $z>z_{a}$ and it is given by the expression (22) in the region $z<z_{a}$. The latter is finite everywhere including the points on the plate.

For points with the proper distances from the brane much larger compared with the AdS curvature radius one has $z \gg z_{a}$. This limit is important from the point of view of the application to the Randall-Sundrum braneworld. To the leading order one has $\left\langle T_{0}^{0}\right\rangle^{(a)} \propto\left(z_{a} / z\right)^{2 \sigma},\left\langle T_{D}^{D}\right\rangle^{(a)} \approx D\left\langle T_{0}^{0}\right\rangle^{(a)} /(D+2 \sigma)$, and the brane-induced VEVs are suppressed by the factor $\exp [2 \sigma(a-y) / \alpha]$. The limit $z \ll z_{a}$ for a fixed $\alpha$ corresponds to points near the AdS boundary $z=0$, with the proper distances from the brane much larger compared with the AdS curvature radius. In this limit to the leading order we have $\left\langle T_{0}^{0}\right\rangle^{(a)} \propto\left(z / z_{a}\right)^{D+2 \sigma},\left\langle T_{D}^{D}\right\rangle^{(a)} \approx-D\left\langle T_{0}^{0}\right\rangle^{(a)} /(2 \sigma)$ and the exponential suppression is by the factor $\exp [(D+2 \sigma)(y-a) / \alpha]$.

\subsection{Two-branes geometry}

In this section we will investigate the $\mathrm{VEV}$ for the energy-momentum tensor in the region between two parallel branes. This VEV is presented in the form

$$
\begin{aligned}
\left\langle 0\left|T_{i}^{k}\right| 0\right\rangle= & \left\langle T_{i}^{k}\right\rangle_{\mathrm{AdS}}+\left\langle T_{i}^{k}\right\rangle^{(j)}-\frac{\alpha^{-D-1} z^{D} \delta_{i}^{k}}{2^{D-1} \pi^{D / 2} \Gamma(D / 2)} \\
& \times \int_{0}^{\infty} d u u^{D-1} \Omega_{j \sigma}\left(u z_{a}, u z_{b}\right) F^{(i)}\left[G_{\sigma}^{(j)}\left(u z_{a}, u z\right)\right],
\end{aligned}
$$

with the functions $F^{(i)}[g(v)]$ from Eqs. (20),(21) and with

$$
\begin{aligned}
\Omega_{a \sigma}(u, v) & =\frac{\bar{K}_{\sigma}^{(b)}(v) / \bar{K}_{\sigma}^{(a)}(u)}{\bar{K}_{\sigma}^{(a)}(u) \bar{I}_{\sigma}^{(b)}(v)-\bar{K}_{\sigma}^{(b)}(v) \bar{I}_{\sigma}^{(a)}(u)}, \\
\Omega_{b \sigma}(u, v) & =\frac{\bar{I}_{\sigma}^{(a)}(u) / \bar{I}_{\sigma}^{(b)}(v)}{\bar{K}_{\sigma}^{(a)}(u) \bar{I}_{\sigma}^{(b)}(v)-\bar{K}_{\sigma}^{(b)}(v) \bar{I}_{\sigma}^{(a)}(u)}, \\
G_{\sigma}^{(a)}(u, v) & =I_{\sigma}(v) \bar{K}_{\sigma}^{(a)}(u)-K_{\sigma}(v) \bar{I}_{\sigma}^{(a)}(u),
\end{aligned}
$$

where the barred notations are defined in (19). In $(23),\left\langle T_{i}^{k}\right\rangle^{(j)}$ is the $\mathrm{VEV}$ of the energy-momentum tensor induced by a single brane located at $y=j$. The cases $j=a$ and $j=b$ provide two equivalent representations for the VEV.

The vacuum force acting per unit surface of the brane at $z=z_{j}$ is determined by the ${ }_{D}^{D}$ - component of the vacuum energy-momentum tensor at this point. The corresponding effective pressures are decomposed as $p^{(j)}=p_{1}^{(j)}+p_{(\mathrm{int})}^{(j)}, j=a, b$, 
where the first term on the right is the pressure for a single brane at $z=z_{j}$ when the second plate is absent. This term is divergent due to the surface divergences and needs an additional renormalization. The term

$$
\begin{aligned}
p_{(\mathrm{int})}^{(j)}= & \frac{\alpha^{-D-1}}{2^{D} \pi^{D / 2} \Gamma(D / 2)} \int_{0}^{\infty} d x x^{D-1} \Omega_{j \sigma}\left(x z_{a} / z_{j}, x z_{b} / z_{j}\right) \\
& \times\left\{\left(x^{2}-\sigma^{2}+2 m^{2} \alpha^{2}\right) B_{j}^{2}-D(4 \xi-1) A_{j} B_{j}-A_{j}^{2}\right\} .
\end{aligned}
$$

is the pressure induced by the presence of the second plate, and can be termed as an interaction force. The effective pressures (25) are negative for Dirichlet scalar and for a scalar with $A_{a}=A_{b}=0$. In these cases the corresponding interaction forces are attractive for all values of the branes separation.

At small distances between the branes, $(b-a) \ll \alpha$, the leading terms are the same as for parallel plates in the Minkowski bulk with $p_{(\mathrm{int})}^{(j)} \propto(b-a)^{-D-1}$. In this limit the interaction forces are repulsive in the case of Dirichlet boundary condition on one plate and non-Dirichlet boundary condition on the another and the forces are attractive for all other cases. For large distances between the branes, $(b-a) \gg \alpha$, one has $p_{(\text {int })}^{(a)} \propto \exp [(D+2 \sigma)(a-b) / \alpha]$ and $p_{(\text {int })}^{(b)} \propto \exp [2 \sigma(a-b) / \alpha]$. With dependence of the values for the coefficients in the boundary conditions, the corresponding forces can be either repulsive or attractive.

The results obtained in this section can be directly applied to the RandallSundrum braneworld model ${ }^{2}$. In the $D$-dimensional generalization of this model the coordinate $y$ in (15) is compactified on an orbifold, $S^{1} / Z_{2}$ of length $L$, with $-L \leqslant y \leqslant L$. The orbifold fixed points at $y=0$ and $y=L$ are the locations of two $(D-1)$-branes. The metric in the Randall-Sundrum model has the form (15) with $e^{-2 y / \alpha} \rightarrow e^{-2|y| / \alpha}$. The action functional for a scalar $\varphi$ has the form

$$
S=\frac{1}{2} \int d^{D} x d y \sqrt{|g|}\left\{g^{i k} \partial_{i} \varphi \partial_{k} \varphi-\left[m^{2}+c_{1} \delta(y)+c_{2} \delta(y-L)+\xi R\right] \varphi^{2}\right\},
$$

with $c_{1}$ and $c_{2}$ being the mass terms on the branes $y=0$ and $y=L$ respectively. The $\mathrm{VEV}$ of the energy-momentum tensor of this scalar is obtained from the formula (23) by an additional factor $1 / 2$. For the coefficients in the boundary conditions one has (see also Ref. 16 for the case $c_{1}=c_{2}=0$ )

$$
\tilde{A}_{a} / \tilde{B}_{a}=-\left(c_{1}+4 D \xi / \alpha\right) / 2, \quad \tilde{A}_{b} / \tilde{B}_{b}=-\left(-c_{2}+4 D \xi / \alpha\right) / 2,
$$

for an untwisted scalar and $\tilde{B}_{a}=\tilde{B}_{b}=0$ (Dirichlet boundary conditions) for a twisted scalar. The energy-momentum tensor in the Randall-Sundrum braneworld for a bulk scalar with zero brane mass terms $c_{1}$ and $c_{2}$ is considered in Ref. 18 . In this paper only a general formula is given for the unrenormalized VEV in terms of the differential operator acting on the Green function. In our approach the part due to the AdS bulk without boundaries is explicitly extracted and in this way the renormalization is reduced to that in the boundary-free AdS spacetime. In addition, we have presented the brane-induced parts in terms of exponentially convergent integrals convenient for numerical calculations. 
We have considered the bulk energy-momentum tensor. For a scalar field on manifolds with boundaries in addition to this part, the energy-momentum tensor contains a contribution located on the boundary (for the expression of the surface energy-momentum tensor in the case of arbitrary bulk and boundary geometries see Ref. 15). The vacuum expectation value of the surface energy-momentum tensor for the geometry of two parallel branes in AdS bulk is evaluated in Ref. 17. It is shown that for large distances between the branes the induced surface densities give rise to an exponentially suppressed cosmological constant on the branes. In particular, in the Randall-Sundrum model the cosmological constant generated on the visible brane is of the right order of magnitude with the value suggested by the cosmological observations. The local vacuum densities in higher-dimensional braneworld models with compact internal subspaces have been investigated in Ref. 10 (for the total vacuum energy in these models see Ref. 19).

\section{Conclusion}

In the present paper we have considered the Casimir densities for a massive scalar field in $(D+1)$-dimensional dS spacetime having the spatial topology $\mathrm{R}^{p} \times\left(\mathrm{S}^{1}\right)^{q}$. Both cases of the periodicity and antiperiodicity conditions along the compactified dimensions are discussed. We have decomposed the VEV of the energy-momentum tensor into the uncompactified dS and topological parts. Since the toroidal compactification does not change the local geometry, in this way the renormalization of the energy-momentum tensor is reduced to that for uncompactifeid $\mathrm{d} \mathrm{S}_{D+1}$.

At early stages of the cosmological expansion the vacuum energy-momentum tensor coincides with the corresponding quantity for a conformally coupled massless field and the topological part behaves like $e^{-(D+1) t / \alpha}$. In this limit the topological part dominates in the VEV. At late stages of the cosmological expansion and for real $\nu$ the topological part is suppressed by the factor $e^{-(D-2 \nu) t / \alpha}$ and the vacuum stresses are isotropic. In the same limit and for pure imaginary $\nu$ the topological terms oscillate with the amplitude going to the zero as $e^{-D t / \alpha}$ for $t \rightarrow+\infty$.

In the second part, we have investigated the VEV of the energy-momentum tensor for a scalar field satisfying Robin boundary conditions on two parallel branes in AdS spacetime. The part of this tensor corresponding to the components on the hyperplane parallel to the brane is proportional to the corresponding metric tensor. The vacuum forces acting on the branes are decomposed into self-action and interaction parts. The interaction forces are given by formula (25) with $j=a, b$ for the brane at $z=z_{a}$ and $z=z_{b}$ respectively. For Dirichlet scalar they are always attractive. In the case of general Robin boundary conditions the interaction forces can be either repulsive or attractive. Moreover, there is a region in the space of Robin parameters in which the interaction forces are repulsive for small distances and are attractive for large distances. This provides a possibility for the stabilization of the interbrane distance by using the vacuum forces. For large distances between the branes, the vacuum interaction forces per unit surface are exponentially suppressed 
by the factor $\exp [2 \sigma(a-b) / \alpha]$ for the brane at $y=a$ and by the factor $\exp [(2 \sigma+$ $D)(a-b) / \alpha]$ for the brane at $y=b$.

\section{Acknowledgments}

The author acknowledges the Organizers of the 5th International Workshop on Astronomy and Relativistic Astrophysics (IWARA2011) and CAPES (Brazil) for a support.

\section{References}

1. N. Arkani-Hamed, S. Dimopoulos and G. Dvali, Phys. Lett. B 429, 263 (1998); Phys. Rev. D 59, 086004 (1999).

2. L. Randall and R. Sundrum, Phys. Rev. Lett. 83, 3370 (1999); L. Randall and R. Sundrum, Phys. Rev. Lett. 83, 4690 (1999).

3. K.A. Milton, The Casimir Effect: Physical Manifestation of Zero-Point Energy (World Scientific, Singapore, 2002); M. Bordag, G.L. Klimchitskaya, U. Mohideen and V.M. Mostepanenko, Advances in the Casimir Effect (Oxford University Press, Oxford, 2009);

4. K.A. Milton, Grav. Cosmol. 9, 66 (2003); E. Elizalde, J. Phys. A 39, 6299 (2006); B. Green and J. Levin, JHEP 11, 096 (2007).

5. A.A. Saharian and M.R. Setare, Phys. Lett. B 659, 367 (2008).

6. S. Bellucci and A.A. Saharian, Phys. Rev. D 77, 124010 (2008).

7. A.A. Saharian, Class. Quantum Grav. 25, 165012 (2008).

8. E.R. Bezerra de Mello and A.A. Saharian, JHEP 12, 081 (2008).

9. A.A. Saharian, Nucl. Phys. B 712, 196 (2005).

10. A.A. Saharian, Phys. Rev. D 73, 044012 (2006); A.A. Saharian, Phys. Rev. D 73, 064019 (2006); A.A. Saharian, Phys. Rev. D 74, 124009 (2006).

11. N.D. Birrell and P.C.W. Davis, Quantum Fields in Curved Space (Cambridge University Press, Cambridge, England, 1982).

12. E. Elizalde, S.D. Odintsov and A.A. Saharian, Phys. Rev. D 79, 065023 (2009); E. Elizalde, S.D. Odintsov and A.A. Saharian, Phys. Rev. D 83, 105023 (2011).

13. P. Breitenlohner and D.Z. Freedman, Ann. Phys. 144, 249 (1982).

14. A. Romeo, A.A. Saharian, J. Phys. A 35, 1297 (2002).

15. A.A. Saharian, Phys. Rev. D 69, 085005 (2004).

16. A. Flachi and D.J. Toms, Nucl. Phys. B 610, 144 (2001).

17. A.A. Saharian, Phys. Rev. D 70, 064026 (2004).

18. A. Knapman and D.J. Toms, Phys. Rev. D 69, 044023 (2004).

19. A. Flachi, J. Garriga, O. Pujolàs and T. Tanaka, JHEP 08, 053 (2003); A. Flachi and O. Pujolàs, Phys. Rev. D 68, 025023 (2003). 